March 26, 2019 0:58 WSPC/INSTRUCTION FILE 'rot'cy'wh-5a

\title{
CYLINDRICAL WORMHOLES: A SEARCH FOR VIABLE PHANTOM-FREE MODELS IN GR
}

\author{
K. A. BRONNIKOV* \\ VNIIMS, Ozyornaya 46, Moscow 119361, Russia; \\ Institute of Gravitation and Cosmology, Peoples' Friendship University of Russia \\ (RUDN University), ul. Miklukho-Maklaya 6, Moscow 117198, Russia; \\ National Research Nuclear University "MEPhI" (Moscow Engineering Physics Institute), \\ Kashirskoe sh. 31, Moscow 115409, Russia \\ kb20@yandex.ru \\ S.V. BOLOKHOV \\ Institute of Gravitation and Cosmology, Peoples' Friendship University of Russia \\ (RUDN University), ul. Miklukho-Maklaya 6, Moscow 117198, Russia; \\ boloh@rambler.ru \\ M.V. SKVORTSOVA \\ Peoples' Friendship University of Russia (RUDN University), \\ ul. Miklukho-Maklaya 6, Moscow 117198, Russia; \\ milenas577@mail.ru
}

The well-known problem of wormholes in general relativity (GR) is the necessity of exotic matter, violating the Weak Energy Condition (WEC), for their support. This problem looks easier if, instead of island-like configurations, one considers string-like ones, among them, cylindrically symmetric space-times with rotation. However, for cylindrical wormhole solutions a problem is the lacking asymptotic flatness, making it impossible to observe their entrances as local objects in our Universe. It was suggested to solve this problem by joining a wormhole solution to flat asymptotic regions at some surfaces $\Sigma_{-}$ and $\Sigma_{+}$on differents sides of the throat. The configuration then consists of three regions, the internal one containing a throat and two flat external ones. We discuss different kinds of source matter suitable for describing the internal regions of such models (scalar fields, isotropic and anisotropic fluids) and present two examples where the internal matter itself and the surface matter on both junction surfaces $\Sigma_{ \pm}$respect the WEC. In one of these models the internal source is a stiff perfect fluid whose pressure is equal to its energy density, in the other it is a special kind of anisotropic fluid. Both models are free from closed timelike curves. We thus obtain examples of regular twice asymptotically flat wormhole models in GR without exotic matter and without causality violations.

Keywords: General relativity, cylindrical symmetry, rotation, wormholes, exact solutions, energy conditions, asymptotic flatness

PACS numbers: 04.20.-q, 04.20.Jb, 04.40.Nr, 04.20.Gz 


\section{Introduction}

The possible existence of traversable Lorentzian wormholes in the Universe is one of the most intriguing predictions of modern physical theories which identify the gravitational field with the properties of space-time geometry. Such objects, being a kind of tunnels in space-time, can make close to each other otherwise distant regions of space as well as different epochs of the Universe evolution, thus acting as time machines. ${ }^{1,2}$ Some researchers consider possible observable effects of wormholes if they really exist in space, ${ }^{3-6}$ see also references therein.

Gravitation is in general an attractive force and tends to focus particle trajectories, whereas in a wormhole geometry some of the paths first approximate but then move away from each other. Such a behavior is a qualitative reason for the necessity of unusual forms of matter, producing repulsion instead of attraction, to support the wormhole geometry. In the framework of general relativity (GR), the necessity of matter violating the weak and null energy conditions (WEC and NEC), called "exotic", or phantom matter, at least in a neighborhood of a wormhole throat (its narrowest place) has been formally established in a number of theorems, ${ }^{1,2,7-9}$ at least in cases where the throat is a compact $2 \mathrm{D}$ surface of finite area. ${ }^{7}$

In theories generalizing GR, a number of examples of wormhole solutions without phantom matter are known, e.g., in the Einstein-Cartan theory, ${ }^{10,11}$ in EinsteinGauss-Bonnet theory, ${ }^{12}$ in multidimensional gravity including brane-world models, ${ }^{13,14}$ etc. In all such cases phantom matter that would be necessary in GR is replaced by geometric or field contributions absent in GR. However, if our interest is in scales, let us say, from meters to kiloparsecs (which can be called macroscopic), it makes sense to adhere to GR since it is this theory that is most reliably confirmed on such scales while its extensions should probably work at very large densities and/or curvatures. It is therefore of great interest to know whether or not wormhole models can be obtained in GR without phantom matter. And in addition, to be observed from distant weakly curved regions of space, such solutions should be asymptotically flat.

Are there known phantom-free wormhole solutions in GR? The answer is yes: there are axially symmetric vacuum solutions such as the Kerr metric with large angular momenta and Zipoy's static solution ${ }^{15}$ as well as their generalizations with electromagnetic and scalar fields. ${ }^{16,17}$ Being asymptotically flat, they, however, contain a ring singularity around a disk that plays the role of a throat, and it appears to be a shortcoming hardly better than the presence of phantom matter. Refs. 18, 19 describe regular wormholes with a source in the form of a nonlinear sigma model, but they are asymptotically NUT-AdS instead of flatness. The wormhole model described in Ref. 20 does not contain phantoms but possesses closed timelike curves and singularities.

The above unpleasant features may be interpreted as manifestations of the socalled topological censorship theorems that forbid unusual topologies assuming that the WEC is observed and that the space-time is asymptotically flat. ${ }^{8,21}$ One of the 
ways to avoid this censorship is to consider configurations like cosmic strings, infinitely stretched in some direction, and the simplest one among them are those with cylindrical symmetry. In the longitudinal $(z)$ direction there is no flat infinity, unless the space-time is completely flat. Static and stationary cylindrically symmetric wormhole solutions were discussed, among many other papers, in Refs. 22-26 (see also references therein). Quite a number of examples of phantom-free cylindrical wormhole solutions in GR have been found.

However, an undesirable feature of almost all cylindrically symmetric solutions is their nonflat asymptotic behavior. Even the Levi-Civita static vacuum solution is only asymptotically flat if it is flat, to say nothing on more complex solutions with matter. To solve this problem and to build asymptotically flat wormhole models, it was suggested ${ }^{23}$ to construct the model space-time from three regions: a central region containing a throat and two Minkowski regions (taken in proper coordinates) attached to this central wormhole solution through some surfaces (cylinders) $\Sigma_{-}$ and $\Sigma_{+}$. Such surfaces inevitably possess some densities and pressures, and their stress-energy tensors (SETs) are determined by jumps of the extrinsic curvature across them. Exotic matter should then be absent both in the internal region and on the surfaces $\Sigma_{-}$and $\Sigma_{+}$. A search for such models began in Refs. 23-26 and is continued here. We will recall some old results and try to construct new models.

The paper is organized as follows. Section 2 presents a general description of stationary cylindrically symmetric space-times, including the notion of cylindrical wormholes, junction conditions on possible discontinuities of the extrinsic curvature, and the WEC and NEC applied to the corresponding surface SETs. In Section 3 we describe some particular wormhole solutions, try to match them to Minkowski regions on both sides of the throat, and test the fulfillment of the energy conditions. Section 4 is a conclusion.

\section{Stationary cylindrical space-times}

\subsection{Basic relations}

Consider the general stationary cylindrically symmetric metric

$$
d s^{2}=\mathrm{e}^{2 \gamma(x)}\left[d t-E(x) \mathrm{e}^{-2 \gamma(x)} d \varphi\right]^{2}-\mathrm{e}^{2 \alpha(x)} d x^{2}-\mathrm{e}^{2 \mu(x)} d z^{2}-\mathrm{e}^{2 \beta(x)} d \varphi^{2},
$$

where $x, z \in \mathbb{R}$ and $\varphi \in[0,2 \pi)$ are the radial, longitudinal and angular coordinates, respectively. The radial coordinate $x$ admits reparametrization $x \rightarrow f(x)$, therefore its range depends on its choice (also called the "gauge") and the geometry itself. The only off-diagonal component $E$ describes rotation that induces a vortex gravitational field characterized by the angular velocity $\omega(x)$ of a congruence of timelike world lines, ${ }^{23,28,29}$

$$
\omega=\frac{1}{2}\left(E \mathrm{e}^{-2 \gamma}\right)^{\prime} \mathrm{e}^{\gamma-\beta-\alpha},
$$

for an arbitrary choice of the coordinate $x$ (the prime denotes $d / d x$ ). Furthermore, if the reference frame is comoving to matter as it moves in the $\varphi$ direction, we the 
SET component $T_{0}^{3}$, is zero, hence (due to the Einstein equations) the Ricci tensor component $R_{0}^{3} \sim\left(\omega \mathrm{e}^{2 \gamma+\mu}\right)^{\prime}=0$, which results in

$$
\omega=\omega_{0} \mathrm{e}^{-\mu-2 \gamma}, \quad \omega_{0}=\text { const. }
$$

Then, according to (2),

$$
E(x)=2 \omega_{0} \mathrm{e}^{2 \gamma(x)} \int \mathrm{e}^{\alpha+\beta-\mu-3 \gamma} d x,
$$

and the Ricci tensor $\left(R_{\mu}^{\nu}\right)$ has the nonzero components

$$
\begin{aligned}
R_{0}^{0} & =-\mathrm{e}^{-2 \alpha}\left[\gamma^{\prime \prime}+\gamma^{\prime}\left(\sigma^{\prime}-\alpha^{\prime}\right)\right]-2 \omega^{2}, \\
R_{1}^{1} & =-\mathrm{e}^{-2 \alpha}\left[\sigma^{\prime \prime}+\sigma^{2}-2 U-\alpha^{\prime} \sigma^{\prime}\right]+2 \omega^{2}, \\
R_{2}^{2} & =-\mathrm{e}^{-2 \alpha}\left[\mu^{\prime \prime}+\mu^{\prime}\left(\sigma^{\prime}-\alpha^{\prime}\right)\right], \\
R_{3}^{3} & =-\mathrm{e}^{-2 \alpha}\left[\beta^{\prime \prime}+\beta^{\prime}\left(\sigma^{\prime}-\alpha^{\prime}\right)\right]+2 \omega^{2}, \\
R_{3}^{0}=G_{3}^{0} & =E \mathrm{e}^{-2 \gamma}\left(R_{3}^{3}-R_{0}^{0}\right),
\end{aligned}
$$

with the following notations:

$$
\sigma=\beta+\gamma+\mu, \quad U=\beta^{\prime} \gamma^{\prime}+\beta^{\prime} \mu^{\prime}+\gamma^{\prime} \mu^{\prime} .
$$

The Einstein equations may be written in two equivalent forms

$$
\begin{aligned}
& G_{\mu}^{\nu} \equiv R_{\mu}^{\nu}-\frac{1}{2} \delta_{\mu}^{\nu} R=-\varkappa T_{\mu}^{\nu}, \quad \text { or } \\
& R_{\mu}^{\nu}=-\varkappa \widetilde{T}_{\mu}^{\nu} \equiv T_{\mu}^{\nu}-\frac{1}{2} \delta_{\mu}^{\nu} T .
\end{aligned}
$$

$\varkappa=8 \pi G$ being the gravitational constant, $R$ the Ricci scalar, and $T$ the trace of the SET. In what follows we mostly work with (8), but it is also necessary to use the constraint equation from (7), which is the first integral of the other Einstein equations, containing only first-order derivatives of the metric functions:

$$
G_{1}^{1}=\mathrm{e}^{-2 \alpha} U+\omega^{2}=-\varkappa T_{1}^{1} .
$$

As is clear from (5), the diagonal components of both Ricci $\left(R_{\mu}^{\nu}\right)$ and Einstein $\left(G_{\mu}^{\nu}\right)$ tensors split into a sum of those for the static metric (i.e., (1) with $E=0$ ) and a contribution from $\omega:^{23}$

$$
\begin{array}{ll}
R_{\mu}^{\nu}={ }_{s} R_{\mu}^{\nu}+{ }_{\omega} R_{\mu}^{\nu}, & { }_{\omega} R_{\mu}^{\nu}=\omega^{2} \operatorname{diag}(-2,2,0,2), \\
G_{\mu}^{\nu}={ }_{s} G_{\mu}^{\nu}+{ }_{\omega} G_{\mu}^{\nu}, & { }_{\omega} G_{\mu}^{\nu}=\omega^{2} \operatorname{diag}(-3,1,-1,1),
\end{array}
$$

where ${ }_{s} R_{\mu}^{\nu}$ and ${ }_{s} G_{\mu}^{\nu}$ are the static parts. Each of the tensors ${ }_{s} G_{\mu}^{\nu}$ and ${ }_{\omega} G_{\mu}^{\nu}$ satisfies the conservation law $\nabla_{\alpha} G_{\mu}^{\alpha}=0$ written in terms of the static metric. It means that ${ }_{\omega} G_{\mu}^{\nu} / \varkappa$ effectively acts as one more SET having exotic properties (for instance, the effective energy density is $\left.-3 \omega^{2} / \varkappa<0\right)$, which is favorable for the existence of wormhole solutions, as has been confirmed by a few examples in Refs. 23, 24, 29. 
Notably, when solving the Einstein equations, it is sufficient to consider their diagonal components; and the only nontrivial off-diagonal component $\left(\begin{array}{l}0 \\ 3\end{array}\right)$ then holds automatically. ${ }^{23}$

\subsection{Wormhole space-times}

The metric (1) is said to describe a wormhole if either (i) the circular radius $r(x)=$ $\mathrm{e}^{\beta(x)}$ has a regular minimum (the corresponding surface $x=$ const is called an $r$-throat) and is large or infinite far from this minimum or (ii) the same is true for the area function $a(x)=\mathrm{e}^{\mu+\beta}$ (its minimum determines an $a$-throat). ${ }^{22,23}$ It is clear that if a wormhole is asymptotically flat on both extremes of the $x$ range, it contains both $r$ - and $a$-throats. On the other hand, it has been shown ${ }^{22,23}$ that in the static case $(E=0)$ such a twice asymptotically flat wormhole should necessarily contain a region where the density of matter is negative. In the general stationary case $(E \neq 0)$ it is not necessary, and in what follows we will assume $E \neq 0$.

Let us, for certainty, use the wormhole definition connected with the circular radius $r(x)$, which looks more evident: moving to smaller $r$, one approaches a wouldbe axis of symmetry $r=0$, but, instead of reaching the axis, meets a minimum of $r$ and its subsequent growth.

Asymptotic flatness requires finite limiting values of $\gamma(x)$ and $\mu(x)$ combined with $\omega=0$, which cannot be achieved according to (3).

To obtain asymptotically flat models, the following method was suggested: ${ }^{23}$ assuming that we have obtained a cylindrical wormhole solution with a non-phantom matter source $T_{\mu}^{\nu}$, cut it on some regular cylinders $\Sigma_{+}\left(x=x_{+}\right)$and $\Sigma_{-}\left(x=x_{-}\right)$ on both sides of the throat and join it there to flat-space regions extending to infinity. Due to discontinuities of the extrinsic curvature on such junction surfaces, they comprise thin shells with certain surface SETs, and it remains to check whether these SETs satisfy the WEC and NEC.

\subsection{Junction surfaces and energy conditions}

Consider a surface $\Sigma\left(x=x_{s}\right)$ separating two regions $\mathbb{D}_{-}\left(x \leq x_{s}\right)$ and $\mathbb{D}_{+}$ $\left(x \geq x_{s}\right)$ with two different metrics of the form (1). Since the metric on $\Sigma$ must be the same, be it calculated from $\mathbb{D}_{-}$or $\mathbb{D}_{+}$, we have the following matching conditions:

$$
[\beta]=0, \quad[\mu]=0, \quad[\gamma]=0, \quad[E]=0,
$$

with the usual notation for discontinuities: for any $f(x),[f]=f\left(x_{s}+0\right)-f\left(x_{s}-0\right)$. The conditions (12) allow us to identify the coordinates $t, z, \phi$ in the whole space. Meanwhile, the choice of radial coordinates in $\mathbb{D}_{+}$and $\mathbb{D}_{-}$may be different, but it is unimportant since the quantities involved in all matching conditions are insensitive to this choice.

The next step is to determine the material content of the surface $\Sigma$ using the Darmois-Israel formalism: ${ }^{31,32}$ in our case of a timelike surface, the SET $S_{a}^{b}$ is 
expressed in terms of the extrinsic curvature $K_{a}^{b}$ as

$$
S_{a}^{b}=(8 \pi G)^{-1}\left[\widetilde{K}_{a}^{b}\right], \quad \widetilde{K}_{a}^{b}:=K_{a}^{b}-\delta_{a}^{b} K_{c}^{c},
$$

where $a, b, c=0,2,3$. The question is whether the surface SET on $\Sigma$ satisfies the WEC whose requirements are

$$
S_{00} / g_{00}=\sigma_{s} \geq 0, \quad S_{a b} \xi^{a} \xi^{b} \geq 0,
$$

where $\sigma_{s}$ is the surface energy density and $\xi^{a}$ an arbitrary null vector on $\Sigma$. The second inequality makes the content of the NEC as a part of the WEC, and taken together, they provide $\sigma_{s} \geq 0$ in any reference frame on $\Sigma$.

It is straightforward to find the following nonzero components of $K_{a b}=\frac{1}{2} \mathrm{e}^{-\alpha} g_{a b}^{\prime}$ on a surface $x=x_{s}$ :

$$
\begin{aligned}
& K_{00}=\mathrm{e}^{-\alpha+2 \gamma} \gamma^{\prime}, \\
& K_{03}=-\frac{1}{2} \mathrm{e}^{-\alpha} E^{\prime}, \\
& K_{22}=-\mathrm{e}^{-\alpha+2 \mu} \mu^{\prime}, \\
& K_{33}=-\mathrm{e}^{-\alpha+2 \beta} \beta^{\prime}+\mathrm{e}^{-\alpha-2 \gamma}\left(E E^{\prime}-E^{2} \gamma^{\prime}\right) .
\end{aligned}
$$

Since $K=g^{a b} K_{a b}=\mathrm{e}^{-\alpha}\left(\beta^{\prime}+\gamma^{\prime}+\mu^{\prime}\right)$, it is easy to obtain the components of $\widetilde{K}_{a b}=$ $K_{a b}-g_{a b} K$, to be further used instead of $S_{a b}=\left[\widetilde{K}_{a b}\right] / \varkappa$ for WEC verification:

$$
\begin{aligned}
& \widetilde{K}_{00}=-\mathrm{e}^{-\alpha+2 \gamma}\left(\beta^{\prime}+\mu^{\prime}\right), \\
& \widetilde{K}_{03}=-\frac{1}{2} \mathrm{e}^{-\alpha} E^{\prime}+E \mathrm{e}^{-\alpha}\left(\beta^{\prime}+\gamma^{\prime}+\mu^{\prime}\right), \\
& \widetilde{K}_{22}=\mathrm{e}^{-\alpha+2 \mu}\left(\beta^{\prime}+\gamma^{\prime}\right), \\
& \widetilde{K}_{33}=\mathrm{e}^{-\alpha+2 \beta}\left(\gamma^{\prime}+\mu^{\prime}\right)+\mathrm{e}^{-\alpha-2 \gamma}\left[E E^{\prime}-E^{2}\left(\beta^{\prime}+2 \gamma^{\prime}+\mu^{\prime}\right)\right] . .
\end{aligned}
$$

From (16) we obtain the condition $\sigma_{s} \geq 0$ in the form

$$
\left[\mathrm{e}^{-\alpha}\left(\beta^{\prime}+\mu^{\prime}\right)\right] \leq 0 \text {. }
$$

Meanwhile, the NEC validity must be proved for any null vector $\xi^{a}$ on $\Sigma$. To cover all null directions on $\Sigma_{ \pm}$, we should take a family of vectors $\xi^{a}$ depending on one parameter, say, $h$, and to try to choose such parameters in our metrics that the conditions (14) will hold for any $h$ from the appropriate range. This proves to lead to rather bulky calculations.

However, the NEC fulfillment may be verified in another way: if in the comoving reference frame the density $\sigma_{s}$ and the pressures $p_{i}$ in mutually orthogonal directions satisfy the inequalities

$$
\sigma_{s} \geq 0, \quad \sigma_{s}+p_{i} \geq 0,
$$

then the WEC holds. A difficulty is that $\Sigma$ is in general not described in a comoving frame.

It is still not necessary to find an explicit transformation to the comoving frame for matter residing on $\Sigma$, which can be an uneasy task. Instead, it is sufficient to find the values of $\sigma_{s}$ and the pressures $p_{z}, p_{\varphi}$ in such a frame as eigenvalues of the 
surface SET and then check whether or not (14) holds. These eigenvalues should be calculated in a local Minkowski (tangent) space, to avoid distortions from the nontrivial metric.

Let us find the tangent-space (triad) components of $\widetilde{K}_{a b}$ using the following orthonormal triad on $\Sigma$ :

$$
e_{(0)}^{a}=\left(\mathrm{e}^{-\gamma}, 0,0\right) ; \quad e_{(2)}^{a}=\left(0, \mathrm{e}^{-\mu}, 0\right) ; \quad e_{(3)}^{a}=\left(E \mathrm{e}^{-\beta-2 \gamma}, 0, \mathrm{e}^{-\beta}\right)
$$

(the parentheses mark triad indices). The triad components $\widetilde{K}_{(m n)}=e_{(m)}^{a} e_{(n)}^{b} \widetilde{K}_{a b}$ turn out to be surprisingly simple and may be represented by the matrix

$$
\left(\widetilde{K}_{(m n)}\right)=\left(\begin{array}{ccc}
-\mathrm{e}^{-\alpha}\left(\beta^{\prime}+\mu^{\prime}\right) & 0 & -\omega \\
0 & \mathrm{e}^{-\alpha}\left(\beta^{\prime}+\gamma^{\prime}\right) & 0 \\
-\omega & 0 & \mathrm{e}^{-\alpha}\left(\gamma^{\prime}+\mu^{\prime}\right)
\end{array}\right) .
$$

The shell matter SET consists of discontinuities of these matrix elements divided by $\varkappa$. The matrix of these discontinuities has the same structure as (20):

$$
\left(\left[\widetilde{K}_{(m n)}\right]\right)=\left(\begin{array}{ccc}
a & 0 & d \\
0 & b & 0 \\
d & 0 & c
\end{array}\right)
$$

and its eigenvalues are easily found as roots of its characteristic equation:

$$
\left(\frac{1}{2}\left(a+c+\sqrt{(a-c)^{2}+4 d^{2}}\right), b, \frac{1}{2}\left(a+c-\sqrt{(a-c)^{2}+4 d^{2}}\right)\right),
$$

The SET in question has the form $S_{(m n)}=\operatorname{diag}\left(\sigma_{s}, p_{z}, p_{\varphi}\right)$, but there is a small problem of which eigenvalues (22) should be identified with particular SET components. To fix it, we notice that in the absence of rotation, that is, if $d=0$, we have $\left(\sigma_{s}, p_{z}, p_{\varphi}\right) \propto(a, b, c)$. Accordingly, we take

$$
\left(\sigma_{s}, p_{z}, p_{\varphi}\right) \propto\left(\frac{1}{2}\left(a+c+\sqrt{(a-c)^{2}+4 d^{2}}\right), b, \frac{1}{2}\left(a+c-\sqrt{(a-c)^{2}+4 d^{2}}\right)\right),
$$

under the assumption $a-c>0$. (Otherwise we must interchange $a$ and $c$.) Then the WEC requirements read

$$
\begin{aligned}
& a+c+\sqrt{(a-c)^{2}+4 d^{2}} \geq 0, \\
& a+c+\sqrt{(a-c)^{2}+4 d^{2}}+2 b \geq 0, \\
& a+c \geq 0 .
\end{aligned}
$$

One can notice that $a$ coincides with the quantity $\varkappa \sigma_{s}$ (17) calculated in the initial noncomoving frame, hence we must also have $a \geq 0$.

\subsection{Minkowski regions}

We are going to use Minkowski space-time regions $\mathbb{M}_{ \pm}$around "internal" wormhole regions. The Minkowski metric should be taken in a rotating reference frame to enable matching with (1) where $E \neq 0$. It can be obtained from the inertial-frame 
metric $d s_{\mathrm{M}}^{2}=d t^{2}-d X^{2}-d z^{2}-X^{2} d \varphi^{2}$ by substituting $\varphi \rightarrow \varphi+\Omega t$, where $\Omega=$ const is the angular velocity:

$$
d s_{\mathrm{M}}^{2}=d t^{2}-d X^{2}-d z^{2}-X^{2}(d \varphi+\Omega d t)^{2} .
$$

The relevant quantities in the notations of (1) are

$$
\begin{array}{ll}
\mathrm{e}^{\alpha}=1, \quad \mathrm{e}^{2 \gamma}=1-\Omega^{2} X^{2}, & \mathrm{e}^{2 \beta}=\frac{X^{2}}{1-\Omega^{2} X^{2}}, \\
E & =\Omega X^{2}, \quad \omega=\frac{\Omega}{1-\Omega^{2} X^{2}} . \\
\gamma^{\prime} & =-\frac{\Omega^{X}}{1-\Omega^{2} X^{2}}, \quad \mu^{\prime}=0, \quad \beta^{\prime}=\frac{1}{X}+\frac{\Omega^{2} X}{1-\Omega^{2} X^{2}} .
\end{array}
$$

This metric is stationary and can be matched to an internal metric at $|X|<1 /|\Omega|$, such that the linear rotational velocity is smaller than $c$.

A good exercise is to verify that direct matching of two Minkowski regions with the metric (27) leads to a thin-shell wormhole with negative density of on-shell matter. We identify the surface $X=X_{0}>0$ that bounds the region $\mathbb{M}_{+}: x \geq X_{0}$ with the surface $X=-X_{0}<0$ that bounds the region $\mathbb{M}_{-}: x \leq-X_{0}$ and obtain

$$
-\varkappa \sigma_{s}=\left[\mathrm{e}^{-\alpha}\left(\beta^{\prime}+\mu^{\prime}\right)\right]=\left[\beta^{\prime}\right]=\frac{2}{\left|X_{0}\right|\left(1-\Omega^{2} X_{0}^{2}\right)}>0,
$$

that is, $\sigma_{s}<0$.

\section{Some particular models}

\subsection{Models with scalar fields}

Minimally coupled scalar fields are described by the Lagrangian

$$
L_{s}=2 \varepsilon g^{\mu \nu} \partial_{\mu} \phi \partial_{\nu} \phi-2 V(\phi)
$$

where $V(\phi)$ is an arbitrary potential, and $\varepsilon= \pm 1$ distinguishes normal (canonical) scalar fields $(\varepsilon=+1)$ and phantom ones $(\varepsilon=-1)$.

In the geometry (1), assuming $\phi=\phi(u)$, the scalar field SET has the form

$$
T_{\mu}^{\nu}(\phi)=\varepsilon \mathrm{e}^{-2 \alpha} \phi^{\prime 2} \operatorname{diag}(1,-1,1,1)+\delta_{\mu}^{\nu} V(\phi) .
$$

There are many exact solutions to the Einstein-scalar field equations with and without a potential, including wormhole ones, ${ }^{23,24}$ but it turns out that the construction with two external Minkowski regions around a wormhole region does not lead to phantom-free models. The corresponding no-go theorem ${ }^{25}$ is valid for any SETs having the property $T_{0}^{0}=T_{3}^{3}$, which is true for scalar fields with any $V(\phi)$ and $\varepsilon$. The theorem is proved using the equation $R_{0}^{0}=R_{3}^{3}$. Choosing the harmonic radial coordinate in the metric (1), specified by the condition

$$
\alpha=\beta+\gamma+\mu,
$$


we obtain the equation $R_{0}^{0}=R_{3}^{3}$ in the Liouville form

$$
\beta^{\prime \prime}-\gamma^{\prime \prime}=4 \omega_{0}^{2} \mathrm{e}^{2 \beta-2 \gamma},
$$

and its solution can be written as

$$
\begin{aligned}
& \mathrm{e}^{\eta}:=\mathrm{e}^{\gamma-\beta}=2 \omega_{0} s(k, x), \\
& s(k, x):=\left\{\begin{array}{lll}
k^{-1} \sinh k x, & k>0, & x \in \mathbb{R}_{+} ; \\
x, & k=0, & x \in \mathbb{R}_{+} ; \\
k^{-1} \sin k x, & k<0, & 0<x<\pi /|k|,
\end{array}\right.
\end{aligned}
$$

A further analysis of WEC requirements shows that ${ }^{25}$ one of them can hold on $\Sigma_{+}$ only if $k>0$ in the internal solution, while on $\Sigma_{-}$it definitely implies $k<0$ (or vice versa). This means that whatever particular solution (with fixed parameters including $k$ ) is taken to describe the internal region, the WEC requirements cannot hold simultaneously on $\Sigma_{+}$and $\Sigma_{-}$.

\subsection{Perfect fluids with $p=w \rho$}

Let us now consider another kind of matter for the internal wormhole region, an isotropic perfect fluid with the equation of state $p=w \rho, w=$ const, so that

$$
\begin{aligned}
& T_{\mu}^{\nu}=\rho \operatorname{diag}(1,-w,-w,-w), \\
& \widetilde{T}_{\mu}^{\nu}=T_{\mu}^{\nu}-\frac{1}{2} \delta_{\mu}^{\nu} T_{\sigma}^{\sigma}=\frac{\rho}{2} \operatorname{diag}(3 w+1, w-1, w-1, w-1),
\end{aligned}
$$

plus the off-diagonal component $T_{3}^{0}=E \mathrm{e}^{-2 \gamma}\left(T_{3}^{3}-T_{0}^{0}\right)$. The conservation law $\nabla_{\nu} T_{\mu}^{\nu}=0$ has the form

$$
p^{\prime}+(\rho+p) \gamma^{\prime}=0 \Rightarrow w \rho^{\prime}+(1+w) \gamma^{\prime}=0 .
$$

(the same as in the static case). For $w \neq 0$ it gives

$$
\rho=\rho_{0} \mathrm{e}^{-\gamma(w+1) / w}, \quad \rho_{0}=\text { const },
$$

and, in terms of the harmonic coordinate $x$ (32), the Einstein equations for the metric (1) can be written as

$$
\begin{aligned}
\mathrm{e}^{-2 \alpha} \gamma^{\prime \prime}+2 \omega^{2} & =\frac{3 w+1}{2} \varkappa \rho, \\
\mathrm{e}^{-2 \alpha} \mu^{\prime \prime} & =\frac{w-1}{2} \varkappa \rho, \\
\mathrm{e}^{-2 \alpha} \beta^{\prime \prime}-2 \omega^{2} & =\frac{w-1}{2} \varkappa \rho, \\
\mathrm{e}^{-2 \alpha}\left(\beta^{\prime} \gamma^{\prime}+\beta^{\prime} \mu^{\prime}+\gamma^{\prime} \mu^{\prime}\right)+\omega^{2} & =w \varkappa \rho .
\end{aligned}
$$

Also recall that $\omega=\omega_{0} \mathrm{e}^{-\mu-2 \gamma}$.

There are exact solutions with the metric (1) and different perfect fluids, ${ }^{33-36}$ but their general discussion is out of the scope of this paper. Let us only make some remarks on the simplest cases. 
First, in the case of dust $(w=0)$ the solution is rather easily obtained since the conservation law (36) in this case leads to $\gamma^{\prime}=0$, hence $\gamma=0$ without loss of generality. Next, a sum of (38) and (40) yields $\beta^{\prime \prime}+\gamma^{\prime \prime}=0$, whence $\beta^{\prime \prime}=0$, and $\beta$ is a linear function that cannot have a minimum, so wormhole solutions cannot be expected. Studies of rotating dust solutions can be found in Refs. 37, 38, 39, containing further bibliography.

Second, the case $w=-1$ corresponds to a cosmological constant, for which solutions are well known and well studied. ${ }^{38,40-42}$ For our discussion it is important that wormhole solutions do exist in this case, ${ }^{23}$ but since, just as for scalar fields, here again $T_{0}^{0}=T_{3}^{3}$, such solutions do not lead to phantom-free models.

\subsection{A model with stiff matter, $p=\rho$}

The special case $w=1$ of a perfect fluid corresponds to maximally stiff matter compatible with causality, in which the velocities of sound and light coincide. This circumstance makes easier a search for exact solutions, including inhomogeneous and wave ones; ${ }^{43}$ one can also mention various applications of stiff matter in theoretical cosmology, see, e.g., the recent papers Refs. 44, 45 and references therein.

Returning to our perfect fluid equations, at $w=1$ from (39) we have

$$
\mu^{\prime \prime}=0 \Rightarrow \mu=m x+\mu_{0}, \quad m, \mu_{0}=\text { const, }
$$

and we put $\mu_{0}=0$ by rescaling the $z$ axis. Then from Eqs. (38) and (40) we obtain

$$
\beta^{\prime \prime}=2 \omega_{0}^{2} \mathrm{e}^{2 \beta-2 \gamma}, \quad \beta^{\prime \prime}+\gamma^{\prime \prime}=2 \varkappa \rho_{0} \mathrm{e}^{2 \beta+2 m x} .
$$

It is not easy to find a general solution to these equations, but a special solution of interest is found by assuming $m=0$ (enabling symmetry under reflections $x \rightarrow-x$ ) and $\gamma \equiv 0$. It then follows

$$
\beta^{\prime \prime}=2 \omega_{0}^{2} \mathrm{e}^{2 \beta}, \quad \varkappa \rho_{0}=\omega_{0}^{2} .
$$

This Liouville equation has three branches of solutions (see (34)), but only in one of them the function $\beta(x)$ has a minimum and is of interest in search for wormhole models. This solution can be written as

$$
\mathrm{e}^{\beta}=\frac{k}{\sqrt{2 \omega_{0}^{2}} \cos (k x)}, \quad k=\text { const }>0, \quad x \in(-\pi / 2, \pi / 2),
$$

where we suppress another integration constant by choosing the zero point of $x$. It remains to find $E$ using the expression (4):

$$
E=\frac{1}{\omega_{0}} \int \frac{k^{2} d x}{\cos ^{2}(k x)}=\frac{k}{\omega_{0}} \tan (k x),
$$

where the integration constant is chosen so that $E(x)$ is an odd function for convenient matching with the exterior metric. Thus the metric is known completely.

It is convenient to pass on to another radial variable, $y=k \tan (k x)$, so that

$$
d x=\frac{d y}{k^{2}+y^{2}}, \quad \mathrm{e}^{2 \beta}=\frac{k^{2}+y^{2}}{2 \omega_{0}^{2}}, \quad E=\frac{y}{\omega_{0}},
$$


and the metric takes the form

$$
d s^{2}=\left(d t-\frac{y}{\omega_{0}} d \varphi\right)^{2}-\frac{d y^{2}}{2 \omega_{0}^{2}\left(k^{2}+y^{2}\right)}-d z^{2}-\left(k^{2}+y^{2}\right) \frac{d \varphi^{2}}{2 \omega_{0}^{2}} .
$$

This solution is regular in the whole range $y \in \mathbb{R}$, but at $y^{2}>k^{2}$ we have $g_{33}>0$, hence the coordinate circles parametrized by $\varphi$ are timelike and violate causality.

We are now almost ready to match this metric to (27) at some surface $y=y_{0}$ identified with some $X=X_{0}$ in (27) according to the conditions (12). However, to obtain $[\gamma]=0$ it is necessary to change the time scale in (48), such that

$$
d t=\sqrt{P} d \tau, \quad P=\text { const }<1
$$

Then we can identify the time $\tau$ on the surfaces $\Sigma_{ \pm}$with $t$ from the external metric and provide $[\gamma]=0$. For the internal metric (48) we then have, instead of the last relation in (47), $E=\sqrt{P} y / \omega_{0}$. (One can verify that this new $E$ is the off-diagonal component $-g_{\tau \phi}$.)

Consider for certainty the surface $\Sigma_{+}\left(y=y_{0}>0, X=X_{0}>0\right)$. The conditions $[\gamma]=0,[E]=0$ and $[\beta]=0$ give, respectively,

$$
P=1-\Omega^{2} X^{2}, \quad \Omega X^{2}=\frac{y \sqrt{P}}{\omega_{0}}, \quad \frac{k^{2}+y^{2}}{2 \omega_{0}^{2}}=\frac{X^{2}}{P} .
$$

where we assume $\omega_{0}>0$ and, without risk of confusion, omit the index "zero" at $X$ and $y$. We notice that there are four independent parameters $\left(P, k, \omega_{0}, y\right)$ in the internal metric (48) after the substitution (49) and two parameters $(\Omega, X)$ in the external metric (27). These six parameters are connected by three equalities (50). Let us choose the following three parameters as independent ones: $X=X_{0}$, having the dimension of length, as a length scale, $y=y_{0}$, and $P$. We then obtain:

$$
\Omega=\frac{\sqrt{1-P}}{X}, \quad \omega_{0}=\frac{\sqrt{P} y}{\sqrt{1-P} X}, \quad k^{2}=y^{2} \frac{1+P}{1-P},
$$

and, as a result, the quantities $a, b, c, d$ from (21)-(24) are expressed as

$$
a=\frac{P^{3 / 2} y-1}{P X}, \quad b=\frac{1-y \sqrt{P}}{X}, \quad c=\frac{P-1}{P X}, \quad d=\frac{\sqrt{P} y-1+P}{X P \sqrt{1-P}} .
$$

The factor $1 / X$ is common and does not affect any inequalities of interest. The condition $a>0$ implies $y>P^{-3 / 2}$. However, since $c<0$, the requirement (26) $a+c \geq 0$ gives a stronger restriction

$$
y \geq \frac{2-P}{P^{3 / 2}}
$$

Then (24) manifestly holds, while (25) requires verification due to $b<0$, An inspection shows that (25) does hold owing to (53).

Thus under the condition (53) the WEC is fulfilled on $\Sigma_{+}$. What changes on $\Sigma_{-}$specified by $X=-X_{0}<0$ and $y=-y_{0}<0$ ? In all parameters (52) the factor $1 / X$ becomes negative. But simultaneously changes the sign of all discontinuities: while on $\Sigma_{+}$we took $[f]=f_{\text {out }}-f_{\text {in }}$ for any quantity $f$, on $\Sigma_{-}$we must take the 
opposite. Therefore, the parameters $a, b, c$ have the same form as in (52) but with $X$ replaced by $|X|$ (recall that we denote, as before, $y=y_{0}>0$ ). For $d=-[\omega]$ we must take into account that, according to (50), $\Omega\left(\Sigma_{-}\right)=-\Omega\left(\Sigma_{+}\right)$, while in the internal solution $\omega\left(\Sigma_{-}\right)=\omega\left(\Sigma_{+}\right)$, therefore, on $\Sigma_{-}$

$$
d \mapsto d_{-}=-\frac{1-P+|y| \sqrt{P}}{|X| P \sqrt{1-P}}
$$

so that $\left|d_{-}\right|>|d|$, which makes it even easier to satisfy the requirement (25). As a result, all WEC requirements are satisfied under the same condition (53), and we obtain a completely phantom-free wormhole model.

It is also important that by $(51)$ we have $y_{0}^{2}<k^{2}$, hence $y^{2}<k^{2}$ in the whole internal region, and there are no closed timelike curves.

\subsection{A model with an anisotropic fluid}

Let us briefly describe one more model, obtained in Ref. 26 with a source in the form of an anisotropic fluid having the SET

$$
T_{\mu}^{\nu}=\rho \operatorname{diag}(1,-1,1,-1) \oplus T_{3}^{0}=-2 \rho E \mathrm{e}^{-2 \gamma},
$$

chosen by analogy with that of a $z$-directed magnetic field in static cylindrical symmetry (which cannot be directly extended to the metric (1) with $E \neq 0$ ). From the conservation law $\nabla_{\mu} T_{1}^{\mu}=0$ it follows ${ }^{\text {a }}$

$$
\rho=\rho_{0} \mathrm{e}^{-2 \gamma-2 \mu}, \quad \rho_{0}=\text { const }>0,
$$

The Einstein equations are solved ${ }^{26}$ using the harmonic radial coordinate (32), and the solution has the form

$$
\begin{gathered}
r^{2} \equiv \mathrm{e}^{2 \beta}=\frac{r_{0}^{2}}{Q^{2}\left(x_{0}^{2}-x^{2}\right)}, \quad \mathrm{e}^{2 \gamma}=Q^{2}\left(x_{0}^{2}-x^{2}\right), \\
\mathrm{e}^{2 \mu}=\mathrm{e}^{2 m x}\left(x_{0}-x\right)^{1-x / x_{0}}\left(x_{0}+x\right)^{1+x / x_{0}}, \\
E=\frac{r_{0}}{2 x_{0}^{2}}\left[2 x_{0} x+\left(x_{0}^{2}-x^{2}\right) \ln \frac{x_{0}+x}{x_{0}-x}\right], \\
x_{0}:=\frac{\left|\omega_{0}\right|}{\varkappa \rho_{0} r_{0}}, \quad Q^{2}:=\varkappa \rho_{0} r_{0}^{2},
\end{gathered}
$$

The introduced constants $x_{0}$ and $Q$ are dimensionless. The solution contains integration constants $\omega_{0}, \rho_{0}, r_{0}$ and $m$, the coordinate $x$ ranges from $-x_{0}$ to $x_{0}$. The circular radius $r \rightarrow \infty$ as $x \rightarrow \pm x_{0}$, thus confirming a wormhole nature of the geometry, but $x= \pm x_{0}$ are curvature singularities, where the Kretschmann invariant behaves as $\left|x_{0}-x\right|^{-4}$.

a A full description of the anisotropic fluid formalism in the metric (1) can be found, e.g., in Ref. 30 . 
To construct an asymptotically flat configuration, we assume $m=0$, making the solution symmetric with respect to the throat $x=0$. Its matching at some $x= \pm x_{s}<x_{0}$ to $\mathbb{M}_{+}$and $\mathbb{M}_{-}$at $X= \pm X_{s}$ leads to the relations

$$
\begin{aligned}
& X=r_{0}, \quad Q^{2}\left(x_{0}^{2}-x^{2}\right)=1-\Omega^{2} X^{2}=: P, \\
& 2 x_{0}^{2} \sqrt{1-P}=2 x x_{0}+\left(x_{0}^{2}-x^{2}\right) \ln \frac{x_{0}+x}{x_{0}-x},
\end{aligned}
$$

where we omit the index $s$ near $x$ without risk of confusion. The condition $[\mu]=0$ makes us change the $z$ scale in $\mathbb{M}_{ \pm}$so that there $-g_{z z}=M^{2}:=\mathrm{e}^{2 \mu\left(x_{s}\right)}$ taken from the internal metric.

It is convenient to use the ratio $y=x_{s} / x_{0}$ and to introduce the notation $L(y)=$ $\ln [(1+y) /(1-y)]$. Then we have

$$
\begin{aligned}
& M=M(y)=(1-y)^{-(1-y) / 2}(1+y)^{-(1+y) / 2}, \\
& P=P(y)=\left(1-y^{2}\right)\left[1-y L(y)-\frac{1}{4}\left(1-y^{2}\right) L^{2}(y)\right] .
\end{aligned}
$$

Using the conditions (57) and these notations, we can write the quantities $a, b, c, d$ used in the WEC requirements (24)-(26) on $\Sigma_{ \pm}$as follows:

$$
\begin{aligned}
& a=\left[-\mathrm{e}^{-\alpha}\left(\beta^{\prime}+\mu^{\prime}\right)\right]=-\frac{1}{P(y)}+\frac{M(y)}{x_{0}^{2}}\left(\frac{y}{1-y^{2}}+\frac{1}{2} L(y)\right), \\
& b=\left[\mathrm{e}^{-\alpha}\left(\beta^{\prime}+\gamma^{\prime}\right)\right]=1, \\
& c=\left[\mathrm{e}^{-\alpha}\left(\gamma^{\prime}+\mu^{\prime}\right)\right]=-\frac{1}{P(y)}+1+\frac{M(y)}{x_{0}^{2}}\left(\frac{y}{1-y^{2}}-\frac{1}{2} L(y)\right), \\
& d=-[\omega]=-\frac{\sqrt{1-P(y)}}{P(y)} \pm \frac{M(y)}{x_{0}^{2}\left(1-y^{2}\right)},
\end{aligned}
$$

where we have ignored the insignificant factor $1 / r_{0}$ appearing in each of them.

The expressions for $a, b, c, d$ depend on two parameters, $x_{0}$ and $y$, and $a, b, c$ are the same on $\Sigma_{+}$and $\Sigma_{-}$, while it turns out that the value of $d$ does not affect the validity of the conditions (24)-(26): actually, if $a>0$ and $a+c>0$, then (25) holds. All quantities in these conditions are dimensionless, and it can be found that $^{26}$

- The condition $0<P(y)<1$, required by construction, holds for $0<y<0.564$ (all numerical estimates are approximate);

- The conditions $a>0$ and $a>c$ hold in quite a large range of $x_{0}$ and $y$, for example, for $x_{0}=0.5, y \in(0.15,0.47)$ and for $x_{0}=0.3, y \in(0.05,0.53)$.

- The condition $a+c>0$ also holds in almost the same range of the parameters, e.g, for $x_{0}=0.5, y \in(0.15,0.38)$ and for $x_{0}=0.3, y \in(0.05,0.51)$.

Thus there is a significant range in the parameter space $\left(x_{0}, y\right)$ in which this asymptotically flat wormhole model completely satisfies the WEC. It can also be verified that $g_{33}<0$ in the internal solution between $\Sigma_{-}$and $\Sigma_{+}$, hence the model does not contain closed timelike curves. 


\section{Conclusion}

Our consideration demonstrates the possible existence of twice asymptotically flat cylindrically symmetric wormholes without exotic matter and without closed timelike curves in general relativity. In stationary cylindrically symmetric space-times it appears possible to obtain a number of exact wormhole-type solutions to the Einstein equations with various material sources (isotropic and anisotropic fluids, scalar and electromagnetic fields, etc.). However, to provide asymptotic flatness is a separate problem, and here, following Ref. 23 , we construct the whole space-time as a union of three regions: a central region with a throat and two Minkowski regions (taken in appropriate rotating reference frames), attached to the central region using the Darmois-Israel formalism with the corresponding junction conditions.

An important task is to find such models where the effective SET on the junction surfaces is not exotic, i.e., does not violate the WEC/NEC. We analyze this property by finding eigenvalues of the extrinsic curvature tensor discontinuities that represent the shell matter SET in its comoving reference frame.

Our analysis shows the existence of non-empty sets of parameters for which the WEC/NEC are indeed respected. Also, the negative sign of $g_{\phi \phi}$ proves the absence of closed timelike curves in the whole space-time under consideration. Moreover, it seems that our models do not violate the topological censorship theorems that restrict the existence of nontrivial space-time topologies assuming that the WEC holds true and the space-time is asymptotically flat. A possible reason is that our cosmic string-like models are not asymptotically flat in the longitudinal direction and are thus not completely asymptotically flat.

An attractive opportunity is that such asymptotically flat configurations, by analogy with cosmic strings, could form loops with a size much larger that their inherent characteristic length parameters, so that cylindrical symmetry could be approximately valid.

In our view, it is of interest to seek new potentially realistic sources for such geometries and to further study their mathematical and physical properties including possible observational effects.

\section{Acknowledgments}

This publication was supported by the RUDN University program 5-100. The work of KB was also performed within the framework of the Center FRPP supported by MEPhI Academic Excellence Project (contract No. 02.a03.21.0005, 27.08.2013).

\section{References}

1. M. Morris, K.S. Thorne, and U. Yurtsever,Phys. Rev. Lett. 611446 (1988).

2. M. Visser, Lorentzian Wormholes: from Einstein to Hawking (AIP, Woodbury, 1995).

3. A. Doroshkevich, J. Hansen, I. Novikov, A. Shatskiy, Int. J. Mod. Phys. D 18, 1665 (2009); arXiv: 0812.0702.

4. T. Harko, Z. Kovacs, F.S.N. Lobo, Phys. Rev. D 79064001 (2009); arXiv: 0901.3926. 
5. A. A. Kirillov, E. P. Savelova, Grav. Cosmol. 1992 (2013).

6. K.A. Bronnikov, K.A. Baleevskikh,. Grav. Cosmol. 2544 (2019), arXiv: 1812.05704

7. D. Hochberg, M. Visser, Phys. Rev. D 564745 (1997); gr-qc/9704082.

8. J.L. Friedman, K. Schleich, and D.M. Witt, Phys. Rev. Lett. 71 (1993) 1486-1489; Erratum: ibid. 75 (1995) 1872; gr-qc/9305017

9. K.A. Bronnikov, S.G. Rubin, Black Holes, Cosmology and Extra Dimensions (World Scientific, Singapore, 2012).

10. K.A. Bronnikov, A.M. Galiakhmetov, Grav. Cosmol. 21283 (2015); arXiv: 1508.01114.

11. K.A. Bronnikov, A.M. Galiakhmetov, Phys. Rev. D 94124006 (2016); arXiv: 1607.07791.

12. H. Maeda, M. Nozawa, Phys. Rev. D 78024005 (2008).

13. K.A. Bronnikov, S.-W. Kim, Phys. Rev. D 67064027 (2003); gr-qc/0212112.

14. K.A. Bronnikov, M.V. Skvortsova, Grav. Cosmol. 22, 316 (2016).

15. D. Zipoy, J. Math. Phys. 7, 1137 (1966).

16. K.A. Bronnikov, J.C. Fabris, Class. Quantum Grav. 14831 (1997); gr-qc/9603037.

17. G. Miranda,T. Matos, arXiv: 1507.02348.

18. E. Ayón-Beato, F. Canfora, J. Zanelli, Phys. Lett. B 752201 (2016).

19. F. Canfora, N. Dimakis, A. Paliathanasis, Phys. Rev. D 96025021 (2017).

20. F. Schein, P. C. Aichelburg, Phys. Rev. Lett. 774130 (1996).

21. G.J. Galloway, E. Woolgar, Class. Quantum Grav. 14 L1-L7 (1997); gr-qc/9609007

22. K.A. Bronnikov, José P.S. Lemos, Phys. Rev. D 79104019 (2009); arXiv: 0902.2360.

23. K.A. Bronnikov, V.G. Krechet, José P.S. Lemos, Phys. Rev. D 87084060 (2013); arXiv: 1303.2993

24. K.A. Bronnikov, V.G. Krechet, Int. J. Mod. Phys. A 31, 1641022 (2016); arXiv: 1509.04665 .

25. K.A. Bronnikov, J. Phys. Conf. Series 675, 012028 (2016); arXiv: 1509.06924.

26. K.A. Bronnikov, V.G. Krechet, arXiv: 1807.03641

27. K.A. Bronnikov, M.V. Skvortsova, Grav. Cosmol. 20171 (2014); arXiv: 1404.5750.

28. V.G. Krechet, Izvestiya Vuzov, Fiz. No 10, 57 (2007)

29. V.G. Krechet, D.V. Sadovnikov, Grav. Cosmol. 15337 (2009); arXiv: 0912.2181.

30. F. Debbasch, L. Herrera, P.R.C.T. Pereira, N.O. Santos, Gen. Rel. Grav. 38, 1825 (2006); gr-qc/0609068.

31. W. Israel, Nuovo Cim. B 48463 (1967).

32. V.A. Berezin, V.A. Kuzmin, I.I. Tkachev, Phys. Rev. D 362919 (1987).

33. K.A. Bronnikov, J. Phys. A, Math. Gen. 12, 201 (1979)

34. N.O. Santos, R.P. Mondaini, Nuovo Cim. B . 7213 (1982)

35. D. Sklavenites, Class. Quantum Grav. 162753 (1999)

36. B.V. Ivanov, Class. Quantum Grav. 193851 (2002); gr-qc/0205023

37. B.V. Ivanov, Class. Quantum Grav. 195131 (2002); gr-qc/0207013

38. H. Stephani, D. Kramer, M.A.H. MacCallum, C. Hoenselaers, E. Herlt, Exact solutions of Einsteins field equations, Cambridge Monographs on Mathematical Physics (Cambridge University Press, 2009).

39. J.B. Griffiths, N.O. Santos, Class. Quantum Grav. 27125004 (2010); arXiv: 1003.3623

40. M.A.H. MacCallum, N.O. Santos, Class. Quantum Grav. 151627 (1998).

41. C. Lanczos, Z. Physik 21, 73 (1924).

42. T. Lewis, Proc. R. Soc.. A 136, 176 (1932).

43. K.A. Bronnikov, J. Phys. A, Math. Gen. 13, 3455 (1980)

44. S.D. Odintsov, V.K. Oikonomou, arXiv: 1711.04571

45. G. Brando, J.C. Fabris, F.T. Falciano, O. Galkina, arXiv: 1810.07860 